\title{
Polynomials that divide many trinomials
}

\author{
by \\ Hans Peter Schlickewei (Ulm) and Carlo Viola (Pisa)
}

1. Introduction. Let

$$
p(X)=a_{k} X^{k}+a_{k-1} X^{k-1}+\ldots+a_{0}
$$

be a polynomial of degree $k>0$ with rational coefficients. We call a polynomial

$$
T(X)=X^{m}+a X^{n}+b
$$

with complex coefficients $a, b$ and with $m>n>0$ a trinomial. In 1965 Posner and Rumsey [2] made the following conjecture:

Suppose that $p(X)$ divides infinitely many trinomials. Then there exist a non-zero polynomial $Q(X)$ of degree $\leq 2$ and a natural number $r$ such that $p(X)$ divides $Q\left(X^{r}\right)$.

In a recent paper [1], this conjecture was shown to be true by Győry and Schinzel. They proved that it suffices to assume that $p$ divides at least

$$
(4 s d)^{s^{6} 2^{180 d}+8 s l}
$$

trinomials with rational coefficients. Here $d$ is the degree of the splitting field $L$ of $p$ over $\mathbb{Q}$. $s$ is the cardinality of the set of places of $L$ consisting of all infinite places and all places induced by the prime ideal factors of the non-zero roots of $p$. Moreover, $l$ is the number of distinct roots of $p$.

It is the purpose of this paper to improve on this result. In fact, we will give an estimate that avoids the parameter $s$ completely and involves only the degree $k$ of the polynomial $p$. We have

Theorem. Let $p(X)$ be a polynomial of degree $k>0$ with rational coefficients which divides more than

$$
2^{44000} k^{1000}
$$

trinomials $T(X)$ as in (1.2) with complex coefficients. Then there exist a non-zero polynomial $Q(X)$ of degree $\leq 2$ with rational coefficients and a natural number $r$ such that $p(X)$ divides $Q\left(X^{r}\right)$. 
We remark that L. Hajdu also improved (1.3) and extended it to the number field case, but his bound depends on $s$ too.

Our proof depends upon a recent result of Schlickewei and Schmidt [3] on polynomial-exponential equations. We conjecture that the bound (1.4) may be replaced by an absolute bound which does not involve the degree of $p$ at all. However, at present this seems to be out of reach.

In a subsequent paper we will deal with the generalization when the trinomials are replaced by $k$-nomials, i.e. the problem stated at the end of the Introduction in [1]. In that wider setting, we will treat also quantitative versions of Theorems $2 \mathrm{~A}$ and $2 \mathrm{~B}$ of $[1]$.

2. A reduction. The following simple lemma will be useful.

Lemma 2.1. Suppose that the trinomial $T(X)=X^{m}+a X^{n}+b$ has $a$ zero $\alpha$ of multiplicity $\geq 3$. Then $\alpha=0$ (and consequently $b=0$ ).

Proof. We have

$$
T^{\prime}(X)=m X^{m-1}+n a X^{n-1}=X^{n-1}\left(m X^{m-n}+n a\right) .
$$

Thus if $\alpha \neq 0$ is a zero of multiplicity $\geq 3$ of $T, \alpha$ is a zero of multiplicity $\geq 2$ of $T^{*}=m X^{m-n}+n a$. But $T^{* \prime}=m(m-n) X^{m-n-1}$. So such an $\alpha \neq 0$ does not exist.

Let $\alpha_{1}, \ldots, \alpha_{l}$ be the distinct zeros of $p$. We partition the set $\left\{\alpha_{1}, \ldots, \alpha_{l}\right\}$ into disjoint classes as follows: two zeros $\alpha_{i}$ and $\alpha_{j}$ belong to the same class if there exists a root of unity $\zeta$ such that $\alpha_{i}=\zeta \alpha_{j}$.

It is clear that if $p(0)=0$ then $\{0\}$ makes up one class.

Proposition 2.2. Let the hypotheses be the same as in the Theorem. Suppose moreover that $p(0) \neq 0$. Then, if $p$ has a double zero $\alpha$, the set of zeros of $p$ lies in a single class. If $p$ does not have a double zero, then its set of zeros splits into at most two distinct classes.

We proceed to deduce the Theorem from Proposition 2.2. First suppose that $p(0)=0$. Then any trinomial $T(X)$ which is divisible by $p(X)$ will be of the shape

$$
T(X)=X^{m}+a X^{n}=X^{n}\left(X^{m-n}+a\right) .
$$

We may conclude that any zero $\alpha \neq 0$ of $p$ is simple and satisfies the equation

$$
\alpha^{m-n}+a=0
$$

Let $L$ be the splitting field of $p$ over $\mathbb{Q}$ and write $G$ for its Galois group. As $p$ has rational coefficients, any $\sigma \in G$ permutes the non-zero roots of $p$. Thus (2.1) implies that $\sigma(a)=a$ for any $\sigma \in G$. We may conclude that $a \in \mathbb{Q}$.

Write $r=\operatorname{lcm}(n, m-n)$ and $t=r /(m-n)$. We put $Q(X)=X(X+$ $\left.a^{t}\right)$. Then obviously $p(X) \mid Q\left(X^{r}\right)$ and $Q(X) \in \mathbb{Q}[X]$, as asserted in the 
Theorem. Thus we may suppose that $p(0) \neq 0$. If $p$ has a double zero, then by Proposition 2.2 there exist an $a \in \mathbb{C}$ and a natural number $r$ such that

$$
\alpha_{i}^{r}=a \quad \text { for } i=1, \ldots, l .
$$

With the same argument as above we get $a \in \mathbb{Q}$. In view of Lemma 2.1 we may conclude that with $r$ from $(2.2)$ and with $Q(X)=(X-a)^{2}$ the assertion of the Theorem is true.

Next suppose that $p$ has only simple zeros. By Proposition 2.2 we may find complex numbers $a$ and $b$ and a natural number $r$ such that any root of $p(X)$ satisfies one of the equations

$$
x^{r}=a \quad \text { or } \quad x^{r}=b .
$$

Again consider the Galois group $G$ of the splitting field $L$ of $p$ over $\mathbb{Q}$. If all the roots of $p$ satisfy a single one of the equations in (2.3), say the first one, we may argue as above and infer that with $r$ from $(2.3)$ and $Q(X)=X-a$ the assertion of the Theorem is true. Otherwise, again since $G$ permutes the roots of $p$, in view of (2.3) we obtain two alternatives: either $\sigma(a)=a$ and $\sigma(b)=b$ for each $\sigma \in G$, or we may conclude that $a$ and $b$ are permuted under $G$.

In the first case $a$ and $b$ are rational numbers. We may take $r$ from (2.3) and $Q(X)=(X-a)(X-b)$ to get the Theorem. In the second case $a$ and $b$ are conjugates over $\mathbb{Q}$ and have degree 2 . Therefore $Q(X)=(X-a)(X-b) \in$ $\mathbb{Q}[X]$ and the Theorem follows with $r$ from $(2.3)$.

The remainder of the paper deals with a proof of Proposition 2.2.

3. Polynomial-exponential equations. We consider equations of the type

$$
\sum_{l=1}^{q} P_{l}(\mathbf{x}) \boldsymbol{\alpha}_{l}^{\mathbf{x}}=0
$$

in variables $\mathbf{x}=\left(x_{1}, \ldots, x_{N}\right) \in \mathbb{Z}^{N}$, where the $P_{l}$ are polynomials with coefficients in a number field $K$ and where

$$
\boldsymbol{\alpha}_{l}^{\mathbf{x}}=\alpha_{l 1}^{x_{1}} \ldots \alpha_{l N}^{x_{N}}
$$

with given $\alpha_{l j} \in K^{*}(1 \leq l \leq q, 1 \leq j \leq N)$. Let $\mathcal{P}$ be a partition of the set $\Lambda=\{1, \ldots, q\}$. The sets $\lambda \subset \Lambda$ occurring in the partition $\mathcal{P}$ will be considered elements of $\mathcal{P}: \lambda \in \mathcal{P}$. Given $\mathcal{P}$, we may consider the system of equations

$$
\sum_{l \in \lambda} P_{l}(\mathbf{x}) \boldsymbol{\alpha}_{l}^{\mathbf{x}}=0 \quad(\lambda \in \mathcal{P}),
$$

which is a refinement of $(3.1)$. Write $\mathfrak{S}(\mathcal{P})$ for the set of solutions $\mathbf{x}$ of $(3.1 \mathcal{P})$ which are not solutions of $(3.1 \mathcal{Q})$ if $\mathcal{Q}$ is a proper refinement of $\mathcal{P}$. 
Given $\mathcal{P}$, set $l \stackrel{\mathcal{P}}{\sim} m$ if $l$ and $m$ lie in the same subset $\lambda$ of $\mathcal{P}$. Let $G(\mathcal{P})$ be the subgroup of $\mathbb{Z}^{N}$ consisting of $\mathbf{z}$ satisfying

$$
\boldsymbol{\alpha}_{l}^{\mathbf{z}}=\boldsymbol{\alpha}_{m}^{\mathbf{z}} \quad \text { for any } l, m \text { with } l \stackrel{\mathcal{D}}{\sim} m .
$$

Write

$$
A_{0}=\sum_{l \in \Lambda}\left(\begin{array}{c}
N+\delta_{l} \\
N
\end{array}\right)
$$

where $\delta_{l}$ is the total degree of the polynomial $P_{l}$. Set

$$
A=\max \left\{N, A_{0}\right\} .
$$

The following proposition will be crucial in the proof of our Theorem.

Proposition 3.1. Suppose $G(\mathcal{P})=\{\mathbf{0}\}$. Then

$$
|\mathfrak{S}(\mathcal{P})|<2^{60 A^{3}} d^{6 A^{2}} \text {. }
$$

This is Theorem 1 of Schlickewei and Schmidt [3].

4. Application to our problem. We are considering trinomials

$$
T(X)=X^{m}+a X^{n}+b .
$$

The hypothesis in Proposition 2.2 says $b \neq 0$. If $a=0$, then the assertion of Proposition 2.2 is trivial. Thus in the sequel we may suppose that $a b \neq 0$. Also, given two trinomials

$$
T_{1}(X)=X^{m_{1}}+a_{1} X^{n_{1}}+b_{1}, \quad T_{2}(X)=X^{m_{2}}+a_{2} X^{n_{2}}+b_{2},
$$

we may suppose without loss of generality that $\left(m_{1}, n_{1}\right) \neq\left(m_{2}, n_{2}\right)$, as otherwise $p(X)$ divides $\left(a_{1}-a_{2}\right) X^{n_{1}}+b_{1}-b_{2}$. And thus the assertion of Proposition 2.2 would follow at once.

Let $\alpha$ be a zero of $p(X)$. Define

$$
\widetilde{p}(X)=a_{k} \alpha^{k} X^{k}+a_{k-1} \alpha^{k-1} X^{k-1}+\ldots+a_{1} \alpha X+a_{0} .
$$

Then $\widetilde{p}(X / \alpha)=p(X)$. Thus, if $\alpha_{1}, \ldots, \alpha_{k}$ are the zeros of $p$, then $\alpha_{1} / \alpha, \ldots$ $\ldots, \alpha_{k} / \alpha$ are the zeros of $\widetilde{p}$. Clearly, in general $\widetilde{p}$ does not have rational coefficients. However, given a trinomial $T$ and defining $\widetilde{T}$ in analogy with $\widetilde{p}$, we see that if $p$ divides $T$ then $\widetilde{p}$ divides $\widetilde{T}$. We remark that our transformation preserves the classes of zeros introduced in Section 2. So it will suffice to prove Proposition 2.2 for $\widetilde{p}$, which has the advantage that $\widetilde{p}(1)=0$.

Let $\alpha$ and $\beta$ be any other zeros of $\widetilde{p}$. If $\widetilde{p}$ divides a trinomial $\widetilde{T}=X^{m}+$ $A X^{n}+B$, we get

$$
\begin{aligned}
1+A+B & =0, \\
\alpha^{m}+A \alpha^{n}+B & =0, \\
\beta^{m}+A \beta^{n}+B & =0 .
\end{aligned}
$$


We may conclude that

$$
\left|\begin{array}{ccc}
1 & 1 & 1 \\
\alpha^{m} & \alpha^{n} & 1 \\
\beta^{m} & \beta^{n} & 1
\end{array}\right|=\alpha^{n}+\beta^{m}+\alpha^{m} \beta^{n}-\alpha^{n} \beta^{m}-\alpha^{m}-\beta^{n}=0 .
$$

The hypothesis of our Theorem together with the reduction from the beginning of this section imply that (4.1) has at least

$$
2^{44000} k^{1000}
$$

solutions $(m, n) \in \mathbb{Z}^{2}$. On the other hand, equation (4.1) is a special instance of the type of equations discussed in Section 3, in fact with six summands, i.e. in the notation of Section 3 with $q=6$. The elements $\alpha, \beta$ may be written as $\alpha_{2} / \alpha_{1}, \alpha_{3} / \alpha_{1}$, where $\alpha_{1}, \alpha_{2}, \alpha_{3}$ are the three zeros of $p$. As $p$ has degree $k, \alpha$ and $\beta$ generate a number field $K$ of degree $\leq k^{3}$.

In our case we have $N=2$ and $\delta_{1}=\ldots=\delta_{6}=0$. Thus we get $A=6$. Therefore, by Proposition 3.1 for any partition $\mathcal{P}$ of $\{1, \ldots, 6\}$ with $G(\mathcal{P})=$ $\{(0,0)\}$ the equation $(4.1 \mathcal{P})$ has not more than $2^{60 \times 6^{3}}\left(k^{3}\right)^{6 \times 6^{2}}$ solutions $(m, n) \in \mathbb{Z}^{2}$. Since the total number of partitions of $\{1, \ldots, 6\}$ does not exceed $6^{6}$, we may conclude that the total set of partitions $\mathcal{P}$ with $G(\mathcal{P})=$ $\{(0,0)\}$ produces less than

$$
2^{18+60 \times 6^{3}} k^{3 \times 6^{3}}<2^{13000} k^{650}
$$

solutions $(m, n) \in \mathbb{Z}^{2}$.

Comparing (4.2) and (4.3) we may infer that there exists a partition $\mathcal{P}$ of the set $\{1, \ldots, 6\}$ with $G(\mathcal{P}) \neq\{(0,0)\}$. We are going to prove that this implies that at least one of $\alpha, \beta, \alpha / \beta$ is a root of unity. It will follow that the three roots $1, \alpha, \beta$ of $\widetilde{p}$ are contained in at most two different classes and this will imply the assertion of Proposition 2.1 if $p$ has only simple zeros.

By a slight abuse of notation we will write $\left\{\alpha^{x}, \beta^{y}, \alpha^{y} \beta^{x}, \alpha^{x} \beta^{y}, \alpha^{y}, \beta^{x}\right\}$ instead of $\{1, \ldots, 6\}$. We proceed to study the possible partitions:

$$
\left\{\alpha^{x}, \beta^{y}\right\}, \quad\left\{\alpha^{y} \beta^{x}, \alpha^{x} \beta^{y}, \alpha^{y}, \beta^{x}\right\} .
$$

Then $G(\mathcal{P})$ among others has the defining relations

$$
\alpha^{y} \beta^{x}=\alpha^{y}, \quad \alpha^{y} \beta^{x}=\beta^{x},
$$

whence $\beta^{x}=1$ and $\alpha^{y}=1$. Thus either $x=y=0$, i.e. $G(\mathcal{P})=\{(0,0)\}$, or one of $\alpha, \beta$ is a root of unity.

$$
\left\{\alpha^{x}, \beta^{y}\right\}, \quad\left\{\alpha^{y} \beta^{x}, \alpha^{x} \beta^{y}\right\}, \quad\left\{\alpha^{y}, \beta^{x}\right\} .
$$

We get

$$
\alpha^{y-x}=\beta^{y-x}, \quad \alpha^{x}=\beta^{y} .
$$


Thus either $y-x=0$ or $\alpha / \beta$ is a root of unity. If $y=x$ then either $x=y=0$ or again $\alpha / \beta$ is a root of unity.

(c)

$$
\left\{\alpha^{x}, \beta^{y}\right\}, \quad\left\{\alpha^{y} \beta^{x}, \alpha^{y}\right\}, \quad\left\{\alpha^{x} \beta^{y}, \beta^{x}\right\} .
$$

We get

$$
\alpha^{y} \beta^{x}=\alpha^{y}, \quad \alpha^{x} \beta^{y}=\beta^{x} .
$$

Thus either $x=0$ or $\beta$ is a root of unity. If $x=0$, then either $y=0$ or again $\beta$ has to be a root of unity. We may conclude that either $G(\mathcal{P})=\{(0,0)\}$ or one of $\alpha, \beta, \alpha / \beta$ is a root of unity.

$$
\left\{\alpha^{x}, \beta^{y}\right\}, \quad\left\{\alpha^{y} \beta^{x}, \beta^{x}\right\}, \quad\left\{\alpha^{x} \beta^{y}, \alpha^{y}\right\} .
$$

This is symmetric to (c).

$$
\left\{\alpha^{x}, \alpha^{y} \beta^{x}\right\}, \quad\left\{\beta^{y}, \alpha^{x} \beta^{y}, \alpha^{y}, \beta^{x}\right\} .
$$

We get

$$
\beta^{y}=\alpha^{y}, \quad \beta^{y}=\beta^{x}
$$

and conclude $x=y=0$ or one of $\beta, \alpha / \beta$ is a root of unity.

$$
\left\{\alpha^{x}, \alpha^{y} \beta^{x}\right\}, \quad\left\{\beta^{y}, \alpha^{x} \beta^{y}\right\}, \quad\left\{\alpha^{y}, \beta^{x}\right\} .
$$

We get

$$
\alpha^{x}=\alpha^{y} \beta^{x}, \quad \beta^{y}=\alpha^{x} \beta^{y}
$$

which implies $x=0$ or $\alpha$ is a root of unity. If $x=0$ then either $y=0$ or again $\alpha$ is a root of unity.

All the partitions containing a subset with two elements are symmetric to the cases treated above or may be treated in a similarly easy way. So we now study partitions with subsets of three elements:

$$
\left\{\alpha^{x}, \beta^{y}, \alpha^{y} \beta^{x}\right\}, \quad\left\{\alpha^{x} \beta^{y}, \alpha^{y}, \beta^{x}\right\} .
$$

We get $\alpha^{x}=\beta^{y}, \alpha^{y}=\beta^{x}$. Hence $\alpha^{x+y}=\beta^{x+y}$. Thus either $x+y=0$ or $\alpha / \beta$ is a root of unity. If $x+y=0$, we use $\beta^{y}=\alpha^{y} \beta^{x}$ and $\alpha^{x} \beta^{y}=\alpha^{y}$. Together with the previous relations we obtain $\beta^{y}=\alpha^{2 y}, \beta^{2 y}=\alpha^{y}$, whence $\beta^{3 y}=\alpha^{3 y}$. Thus either $y=0$ (and therefore also $x=0$ ), or $\alpha / \beta$ is a root of unity.

$$
\left\{\alpha^{x}, \beta^{y}, \alpha^{x} \beta^{y}\right\}, \quad\left\{\alpha^{y} \beta^{x}, \alpha^{y}, \beta^{x}\right\} .
$$

Then $\alpha^{x}=\beta^{y}, \alpha^{x}=\alpha^{x} \beta^{y}$. Thus either $y=0$ or $\beta$ is a root of unity. If $y=0$ then either $x=0$ or $\alpha$ is a root of unity.

$$
\left\{\alpha^{x}, \alpha^{y} \beta^{x}, \alpha^{x} \beta^{y}\right\}, \quad\left\{\beta^{y}, \alpha^{y}, \beta^{x}\right\} .
$$

We get $\beta^{y}=\alpha^{y}, \beta^{y}=\beta^{x}$. Either $y=0$ or $\alpha / \beta$ is a root of unity. If $y=0$ then either $x=0$ or $\beta$ is a root of unity.

All other cases are symmetric to the ones treated above or at least equally easy. Altogether we have shown that if there exists a partition $\mathcal{P}$ 
with $G(\mathcal{P}) \neq\{(0,0)\}$ then at least one of $\alpha, \beta, \alpha / \beta$ is a root of unity. So Proposition 2.2 follows if $p$ has only simple roots.

We next assume that $p$ has a double root $\alpha$. We may choose our transformation $p \mapsto \widetilde{p}$ such that 1 is a double root of $\widetilde{p}$. Let $\beta$ be any other root of $\widetilde{p}$. Then given a trinomial $\widetilde{T}=X^{m}+A X^{n}+B$ we get $\widetilde{T}(1)=\widetilde{T}^{\prime}(1)=\widetilde{T}(\beta)=0$. Thus

$$
\left|\begin{array}{ccc}
1 & 1 & 1 \\
m & n & 0 \\
\beta^{m} & \beta^{n} & 1
\end{array}\right|=(n-m)+m \beta^{n}-n \beta^{m}=0 .
$$

This is an equation of the type considered in Section 3 . Here $N=2, \delta_{1}=$ $\delta_{2}=\delta_{3}=1, A=9$, and as $\beta$ is the quotient of two roots $\alpha_{i}, \alpha_{j}$ of $p$, it has degree $\leq k^{2}$. With our reductions we see that we are only interested in solutions $(m, n) \in \mathbb{Z}^{2}$ such that no subsum in (4.4) vanishes. Thus for $\mathcal{P}=\{1,2,3\}$ Proposition 3.1 says that (4.4) has less than

$$
2^{60 \times 9^{3}}\left(k^{2}\right)^{6 \times 9^{2}}<2^{44000} k^{1000}
$$

solutions $(m, n) \in \mathbb{Z}^{2}$, provided that $G(\mathcal{P})=\{(0,0)\}$. On the other hand, the hypothesis of the Theorem guarantees that we have at least $2^{44000} k^{1000}$ solutions $(m, n) \in \mathbb{Z}^{2}$. We may infer that $G(\mathcal{P}) \neq\{(0,0)\}$. In our case the defining relations for $G(\mathcal{P})$ are

$$
\beta^{x}=\beta^{y}=1 .
$$

As $G(\mathcal{P}) \neq\{(0,0)\}$, this implies at once that $\beta$ is a root of unity. Therefore the two zeros 1 and $\beta$ of $\widetilde{p}$ lie in the same class. This proves Proposition 2.2 if $p$ has a double root.

\section{References}

[1] K. Győry and A. Schinzel, On a conjecture of Posner and Rumsey, J. Number Theory 47 (1994), 63-78.

[2] E. C. Posner and H. Rumsey, Jr., Polynomials that divide infinitely many trinomials, Michigan Math. J. 12 (1965), 339-348.

[3] H. P. Schlickewei and W. M. Schmidt, On polynomial-exponential equations, II, to appear.

Abteilung Mathematik II

Universität Ulm

Helmholtzstrasse 18

89081 Ulm, Germany

E-mail: hps@mathematik.uni-ulm.de
Dipartimento di Matematica Università di Pisa Via Buonarroti 2 56127 Pisa, Italy E-mail: viola@dm.unipi.it 